\title{
Bridging the Gap with Telemedicine: Community Emergency Department to Tertiary Care Pediatric Intensive Care
}

\author{
Mark L Waltzman ${ }^{1 *}$, Michael C Monuteaux ${ }^{1}$, Monica Kleinnman ${ }^{2}$, Daniel Nigrin ${ }^{3}$ and Karen Gruskin ${ }^{1}$ \\ ${ }^{1}$ Division of Emergency Medicine, Boston Children's Hospital, 300 Longwood Ave, Boston, MA 02115, USA \\ ${ }^{2}$ Division of Critical Care Medicine, Boston Children's Hospital, 300 Longwood Ave, Boston, MA 02115, USA \\ ${ }^{3}$ Division of Endocrinology, Boston Children's Hospital, 300 Longwood Ave, Boston, MA 02115, USA
}

\section{Introduction}

Children account for $22 \%$ of annual United States (US) emergency department visits [1], and require specialized attention due to physiologic, anatomic, emotional and therapeutic differences compared to adults [2]. The current US health care system is often ill-prepared for these differences. Only $7 \%$ of hospitals are fully equipped for pediatric emergencies, and less than $9 \%$ consistently have a pediatrician on-call [1]. The presence of pediatric-specific training has been identified as a positive indicator for improved outcomes, specifically for patients with highly acute conditions, such as shock. In an observational study of 1422 children with signs of shock (abnormal capillary refill, tachycardia, and/ or hypotension) who were transferred from a community hospital setting to a tertiary care pediatric facility, the authors found that early reversal of shock in the community hospital and use of Pediatric Advanced Life Support/Advanced Pediatric Life Support (PALS/APLS) interventions were associated with a decrease in mortality and morbidity (permanent neurologic dysfunction) regardless of underlying etiology (eg: trauma, sepsis) [3]. However, covering gaps in pediatric care is costly and difficult. Increasing access to pediatric specialists is impractical given the lack of sufficient numbers and volume to support their coverage in many areas. Development and maintenance of skills for physicians who infrequently manage critically ill children is challenging.

The published data on telemedicine and pediatric critical care transfers has been primarily focused on use from rural to urban centers, describing initial experiences and adherence to standardized treatment guidelines [4]. There is little data on the utilization of tele-medicine services to assist in the evaluation, stabilization and ultimately the transport modality (air vs. specialized critical care transport team vs advanced life support $v s$. basic life support) of critically ill patients from a community hospital setting to a tertiary care pediatric intensive care unit within a local geographic area or a local network of an academic/ tertiary care "hub" and community "spoke" hospital(s). Understanding the feasibility, acceptance, and effectiveness of using telemedicine for critically ill children in the community ED will be useful in the future evaluation of policy, procedures, guidelines and management for the use of telemedicine, as well as understanding the potential for decreasing the total medical expenditures for caring for these children.

\section{Objective}

Our objective was to evaluate the implementation of a telemedicine program that provided a direct link between community hospital emergency department (ED) providers and tertiary pediatric intensive care unit (ICU) specialists. We described decision-making around the mode of transport, and the care of this population by the physician team at both locations.

\section{Materials and Methods}

\section{Design and Setting}

This was a cross-sectional survey study conducted at two community hospital EDs utilizing a mobile tele-medicine system (InTouch Health ${ }^{n x}$ ) with a connection to the intensive care unit of a free standing, pediatric tertiary care academic hospital from 9/2013$8 / 2015$. The tertiary care hospital serves as a major referral center for New England. The two community hospitals (hospitals A \& B) provide pediatric services to a combined 42,000 patients per year with transfer rate for subspecialty or higher level of care of $1-3 \%$ per year. These community hospitals are staffed by general pediatricians and pediatric emergency medicine specialty trained physicians employed by the tertiary care hospital and through an affiliation agreement, contracted to provide pediatrics care in the community hospital. At community hospital site A, there is $24 / 7$ pediatric ED coverage and at site B there is $12 / 7$ pediatric emergency coverage (although an in-house pediatrician is available 24/7 for consultations). When the pediatrician is not treating patients in the ED, a general emergency medicine physician treats pediatric patients. This study was performed as a quality improvement project and was approved by the Hospital's Institutional Review Board.

\section{Selection of Patients}

All children birth to 21 years (at site A) and birth to 18 years (at site B) who were evaluated from September 2013 through August 2015 at the community hospitals and in need of ICU-level care were eligible for telemedicine intervention. Physicians who utilized telemedicine for these patients were enrolled.

*Correspondence to: Mark L. Waltzman, Division of Emergency Medicine, Boston Children's Hospital, 300 Longwood Ave, Boston, MA 02115, USA, Tel: +(617) 355-6624, E-mail: Mark.waltzman@childrens.harvard.edu

Key words: Telemedicine; telehealth; technology; utilization; decision support

Received: May 02, 2018; Accepted: May 25, 2018; Published: May 29, 2018 


\section{Procedures and Measures}

Upon identification of a pediatric patient in need of ICU-level care, the community hospital ED placed a call to a dedicated $24 / 7$ communications center staffed with emergency medical technicians (EMTs). The EMTs obtained the demographic data and contacted the pediatric intensivist at the tertiary care hospital. Requests for telemedicine connection were made to the ICU physician and a secure link with the community ED was established. The mobile ED system had a monitor attached to the camera allowing the ED physician, nursing staff, and patient/family to see the ICU physician, while the ICU physician controlled the camera that had full pan, zoom, and tilt capabilities in order to evaluate the patient and "scene." In order to facilitate private communication between providers, a secured audio connection was used between the ED and ICU physicians.

Surveys were obtained from the community ED and ICU providers who utilized the telemedicine system to measure their attitude towards and assessment of the technology. The providers were sent an email inviting them to participate. A link to a web-based survey was included in the email. The survey data were collected and managed using Research Electronic Data Capture (REDCap) electronic data capture tools hosted at Boston Children's Hospital [5]. REDCap is a secure, web-based application designed to support data capture for research studies, providing 1 ) an intuitive interface for validated data entry; 2) audit trails for tracking data manipulation and export procedures; 3 ) automated export procedures for seamless data downloads to common statistical packages; and 4) procedures for importing data from external sources [6].

All surveys were conducted via email and for those surveys not completed within 2 weeks, email and phone calls were made to the providers by the PI or the telemedicine team, weekly for 2 additional weeks. The surveys solicited physicians' evaluations of the technology's ability to facilitate communication, clinical assessment and decision making, as well as whether the technology resulted in any changes in treatment plans and transport modality. All responses were captured on a five-point Likert scale.

\section{Statistical Analyses}

Demographic characteristics of the patient sample were summarized with frequencies and proportions for categorical variables and medians with interquartile ranges for continuous variables. Physician survey responses were presented with frequencies and proportions. Percent agreement between matched community and ICU physician pairs on survey items relating to clinical communication and decision making were calculated.

\section{Results}

As shown in Table 1, fifty-three patients were enrolled, ranging from 2-11 years. Respiratory and neurologic conditions were the most frequent conditions where telemedicine was utilized. Seventytwo percent of all transports were performed by a dedicated pediatric critical care transport team.

The telemedicine technology was deemed easy to use and the interaction between providers was reported to be favorable (Tables 2 and 3). The ICU providers felt it was "very easy/easy" to assess the patient's condition via telemedicine in $57 \%$ of cases. Community providers felt that telemedicine impacted their decision for mode of transport $33 \%$ of the time, versus $18 \%$ among the ICU providers. The use of telemedicine resulted in in 6 changes in transport modality with
Table 1. Demographic and clinical characteristics of patients for whom Tele Connect was used.

\begin{tabular}{|c|c|}
\hline Demographic and clinical Characteristics & $\mathbf{n}=\mathbf{5 3}$ \\
\hline Age (years) & $4[2,11]$ \\
\hline Gender (male) & $35(66)$ \\
\hline & \\
\hline Cardiac Arrest & $1(2)$ \\
\hline DKA & $2(4)$ \\
\hline Ingestion & $2(4)$ \\
\hline Neurologic & $18(34)$ \\
\hline Respiratory & $25(47)$ \\
\hline Sepsis & $5(9)$ \\
\hline & \\
\hline Advanced Life support & $7(13)$ \\
\hline Critical Care Transport Team & $38(72)$ \\
\hline Med Flight & $5(9)$ \\
\hline Other & $3(6)$ \\
\hline Values in table represent median [interquartile range] or frequency (percent)
\end{tabular}

Table 2. Referring providers' attitudes toward Tele Connect implementation ( $\mathrm{n}=27)$

\section{Survey Item}

\begin{tabular}{|l|l|} 
Response Description & Frequency (percent)
\end{tabular}

Interaction with the MICU physician

via the telemedicine connection?

Compared with using the phone, to what

degree did the telemedicine connection

contribute to your assessment of the

patient's condition?

Did this method of collaboration change

the management of the patient?

Did this method of collaboration change

the transport modality of the patient?

Overall, to what extent do you feel the

telemedicine connection aided your decision-making?

The telemedicine system is easy to use.

Compared with the phone, to what

degree did telemedicine contribute

to your assessment of the patient's condition?

Did this method of collaboration change the management of the patient?

Did this method of collaboration change the transport modality of the patient?

Overall, to what extent do you feel

telemedicine aided your decisionmaking?

\begin{tabular}{|c|c|}
\hline Rerponse Description & Frequency (percent) \\
\hline A lot/Quite a bit & $22(85)$ \\
\hline A lot/Quite a bit & $7(26)$ \\
\hline A lot/Quite a bit & $2(7)$ \\
\hline $\begin{array}{r}\text { To a large extent/To a } \\
\text { moderate extend }\end{array}$ & $1(4)$ \\
\hline $\begin{array}{r}\text { Strongly agree/Agree } \\
\text { A lot/Quite a bit }\end{array}$ & $22(85)$ \\
\hline $\begin{array}{r}\text { A lot/Quite a bit } \\
\text { moderate extend }\end{array}$ & $7(26)$ \\
\hline $\begin{array}{l}\text { A lot/Quite a bit } \\
\text { a large extent/To a }\end{array}$ & $2(7)$ \\
\hline
\end{tabular}

3 being downgraded from Critical Care Transport to local Advanced Life Support. When considering whether telemedicine resulted in any change in management, $41 \%$ of referring providers (Table 2) and $55 \%$ of ICU providers (Table 4) found at least some benefit. When queried as to whether telemedicine aided in decision making to any degree, $74 \%$ of referring providers and $78 \%$ of MICU providers found value in the technology. There was a 70\% agreement among ED and ICU providers that the implementation of telemedicine improved their assessment of patients over telephone consultation alone and this collaboration resulted in a change in patient management (Tables 3, 5-7).

\section{Discussions}

Overall, our findings support the additional value of the video component of using telemedicine to a traditional telephone conversation between a community hospital emergency department and the pediatric intensive care for assisting in the management and transfer of critically ill children in a community ED. In general, the use of telemedicine was regarded favorably by both the emergency 
Table 3: Referring providers' attitudes toward TeleConnect implementation ( $\mathrm{n}=27)$.

\section{Survey Item}

Compared with the phone, to what degree did telemedicine contribute to your assessment of the patient's condition?

Did this method of collaboration change the management of the patient?

Did this method of collaboration change the transport modality of the patient?

Overall, to what extent do you feel telemedicine aided your decision-making?

\begin{tabular}{|c|c|}
\hline Not at all & A little \\
\hline $11(41)$ & $4(15)$ \\
\hline Not at all & A little \\
\hline $16(59)$ & $4(15)$ \\
\hline Not at all & A little \\
\hline $18(67)$ & $1(4)$ \\
\hline Not at all & Little extent \\
\hline $7(26)$ & $10(37)$ \\
\hline
\end{tabular}

Response Description

\begin{tabular}{|c|c|c|}
\hline Somewhat & Quite a bit & A lot \\
\hline $5(19)$ & $1(4)$ & $6(22)$ \\
\hline Somewhat & Quite a bit & A lot \\
\hline $5(19)$ & $0(0)$ & $2(7)$ \\
\hline Somewhat & Quite a bit & A lot \\
\hline $7(26)$ & $1(4)$ & $0(0)$ \\
\hline Some extent & Mod. extend & Large extent \\
\hline $6(22)$ & $3(11)$ & $1(4)$ \\
\hline
\end{tabular}

Table 4. ICU providers' attitudes toward TeleConnect implementation ( $\mathrm{n}=49)$.

\begin{tabular}{|c|c|c|}
\hline Survey Item & Response Description & Frequency (percent) \\
\hline Interaction with remote physician via the telemedicine connection? & Very easy / Easy & $38(78)$ \\
\hline $\begin{array}{l}\text { Interaction with the patient and family at the remote hospital via the telemedicine } \\
\text { connection? }\end{array}$ & Very easy / Easy & $20(63)$ \\
\hline Assessing the patient's condition via the telemedicine connection? & Very easy / Easy & $28(57)$ \\
\hline $\begin{array}{l}\text { Compared with using the phone, to what degree did the telemedicine connection } \\
\text { contribute to your assessment of the patient's condition? }\end{array}$ & A lot / Quite a bit & $17(35)$ \\
\hline Did this method of collaboration change the management of the patient? & A lot / Quite a bit & $9(21)$ \\
\hline Did this method of collaboration change the transport modality of the patient? & A lot / Quite a bit & $5(12)$ \\
\hline Overall, to what extent do you feel the telemedicine connection aided your decision-making? & To a large extent / To a moderate extend & $14(29)$ \\
\hline $\begin{array}{l}\text { Compared with the phone, to what degree did telemedicine contribute to your } \\
\text { assessment of the patient's condition? }\end{array}$ & A lot / Quite a bit & $17(35)$ \\
\hline Did this method of collaboration change the management of the patient? & A lot / Quite a bit & $9(21)$ \\
\hline Did this method of collaboration change the transport modality of the patient? & A lot / Quite a bit & $5(12)$ \\
\hline Overall, to what extent do you feel telemedicine aided your decision-making? & To a large extent / To a moderate extend & $14(29)$ \\
\hline
\end{tabular}

Table 5. ICU providers' attitudes toward TeleConnect implementation $(n=49)$.

\section{Survey Item}

Compared with the phone, to what degree did telemedicine contribute to your assessment of the patient's condition?

Did this method of collaboration change the management of the patient?

Did this method of collaboration change the transport modality of the patient?

Overall, to what extent do you feel telemedicine aided your decision-making?

\begin{tabular}{|c|c|}
\hline Not at all & A little \\
\hline $3(6)$ & $11(23)$ \\
\hline Not at all & A little \\
\hline $16(37)$ & $10(23)$ \\
\hline Not at all & A little \\
\hline $33(79)$ & $2(5)$ \\
\hline Not at all & Little extent \\
\hline $6(13)$ & $10(21)$ \\
\hline
\end{tabular}

Response Description

\begin{tabular}{|c|c|c|}
\hline Somewhat & Quite a bit & A lot \\
\hline $17(35)$ & $4(8)$ & $13(27)$ \\
\hline Somewhat & Quite a bit & A lot \\
\hline $8(19)$ & $3(7)$ & $6(14)$ \\
\hline Somewhat & Quite a bit & A lot \\
\hline $2(5)$ & $1(2)$ & $4(10)$ \\
\hline Some extent & Mod. extend & Large extent \\
\hline $18(38)$ & $9(19)$ & $5(10)$ \\
\hline
\end{tabular}

Table 6. Agreement between referring and ICU providers on attitudes toward TeleConnect implementation $(\mathrm{n}=23)$.

\section{Survey Item}

Compared with the phone, to what degree did telemedicine contribute to your assessment of

the patient's condition?

Did this method of collaboration change the management of the patient?

Did this method of collaboration change the transport modality of the patient?

Overall, to what extent do you feel telemedicine aided your decision-making?

Percent Agreement

\begin{tabular}{l}
$70 \%$ \\
\hline $70 \%$ \\
\hline $80 \%$ \\
\hline $65 \%$ \\
\hline
\end{tabular}

Table 7. Referring providers' attitudes toward TeleConnect implementation $(n=27)$.

\begin{tabular}{|l|c|}
\hline Survey Item & Response Description \\
\hline Interaction with the MICU physician via the telemedicine connection? & Very easy / Easy \\
\hline $\begin{array}{l}\text { Compared with using the phone, to what degree did the telemedicine connection } \\
\text { contribute to your assessment of the patient's condition? }\end{array}$ & A lot / Quite a bit \\
\hline Did this method of collaboration change the management of the patient? & A lot / Quite a bit \\
\hline Did this method of collaboration change the transport modality of the patient? & A lot / Quite a bit \\
\hline Overall, to what extent do you feel the telemedicine connection aided your decision-making? & To a large extent / To a moderate extend \\
\hline The telemedicine system is easy to use. & Strongly agree / Agree \\
\hline
\end{tabular}

department and critical care physicians. They both found the system easy to use and the interactions were favorable, but there was some discordance between the two types of physicians around the overall usefulness of the system. Emergency providers are well trained in the management of critically ill patients, and they reported less benefit over all in the system; however, when utilized, there was a change in the management in the patient in $67 \%$ of cases which demonstrated a difference between perception and reality. The critical care physicians reported use of the system more favorably. Telemedicine gave them the ability to visualize the patient in real time, allowing them to better assess and make patient specific recommendations on critical care management. In one instance, due to transport delays secondary to 
weather, the ability to co-manage a critically ill 2 year old patient, who required constantly fine-tuning of the ventilatory support, likely resulted in the child's life being saved by the use of this technology.

Successful uses of various telemedicine services have been well documented and have demonstrated positive outcomes. Services including remote interpretation of radiology imaging for the evaluation of head trauma to interventions for improvement in quality of life for patients suffering from chronic obstructive pulmonary disease have experienced wide successes $[7,8]$. Telemedicine as a modality for remote consultations, typically linking a remote center with a specialist at an academic center, can occur either as a delayed review of data (such as echocardiograms or fundoscopic images to evaluate for retinopathy of prematurity) or in real-time via audio-video linkages. These consultations are helpful in the acute and chronic management of illnesses $[9,10]$. In emergency medicine, the technology has been utilized to evaluate patients for transport and has allowed for a more appropriate utilization of these scarce and costly resources [11].

Expansion of tele-health services continue as comfort and acceptance of the technology grows and financial pressures on health care systems and providers expand. Clinicians are asked to see more patients, in less time, but also in a manner that is patient-centered. In order to expand availability, providers and patients will need to embrace alternative modes of care delivery. The in-person, face-to-face encounter may become less frequent as the adoption of "virtual visits" become more common. Emergency tele-psychiatry, for example, has been shown to be a cost affective modality to provide needed care with a sparse resource [12]. The remote provision of health care, where providers and patients are not in the same location, promotes the concept of the patients receiving the right care, at the right time, at the right place, and in the manner they consider right for them; while providers can be compensated for their time and expertize in a manner that is cost effective [13]. The Federal Government has enacted legislation to allow for the credentialing of physicians engaged in telemedicine at remote locations; however individual States have not necessarily followed suit, which has led to obstacles and confusion among providers and health care systems. Additionally, third party payors are not uniform in reimbursement for telemedicine services, which leads to additional challenges in developing unique models of care.

\section{Limitations}

Despite perceived ease of use and demonstrated clinical value, utilization was lower than expected. The majority of the clinicians in the community hospital setting underwent training just prior to rapid social expansion/utilization of video technology; as trainees matriculate, their comfort level with the technology may result in higher acceptance and adaptation. Additional limitations in utilization included issues around the physical design of the emergency departments. The technology was not always readily available at the bedsides most typically used for critically ill pediatric patients due to space constraints; this led to work flow issues resulting from moving the equipment to the bedside. Staff did not have the visual cue of having the telemedicine equipment in the room, reinforcing its availability and use. There was a large number of staff to train in the program in which there was a relatively low overall volume of critically ill patients, therefore the system's use was not regularly reinforced. Anecdotally, staff indicated a need to focus on direct patient care in these critical situations and did not have the resources to bring the technology to the bedside. The critical care specialist often had competing demands as they were working clinically at their local institution while receiving the request for tele-medicine consultations, and at times could not be spared from their clinical duties to participate in the link, which resulted in missed remote-care opportunities. These limitations are common in the adoption of tele-medicine and not unique to our study. In additional to issues with technically challenged staff and resistance to change, cost, reimbursement, and patient specific factors such as age and level of education have been identified as primary barriers to successful implementation [14].

\section{Conclusions}

Adaption to changes in communication can be challenging. Changes in the culture of care as well as in utilization of novel technologies pose challenges in individual and systematic work flows. In this study we demonstrated that despite a change in management of critically ill patients on the side of the intensive care physicians, referring clinicians did not perceive the same benefit and adoption of the technology was challenging. Greater details on the change in patient care in order to provide detailed feedback training is crucial to affecting adoption and culture changes in the use of telemedicine to support real-time support of the provision of pediatric critical care. Additionally, greater adoption will require change to work flows, resources and ultimately facilities making the use of the technology more integrated and streamlined.

\section{Funding}

This project was funding by the Boston Children's Hospital Provider-Payor Quality Initiative.

\section{References}

1. Schappert S, Bhuiya F (2012) National Health Statistics Survey: Availability of pediatric services and equipment in emergency departments: United States. Centers Dis Control Prev 47: 1-21.

2. Institute of Medicine Committee on the Future of Emergency Care in the U.S (2006) Health Care System. Emergency Care for Children: Growing Pains. Washington, DC: National Academy Press.

3. Carcillo JA, Kuch BA, Han YY, Day S, Greenwald BM, et al. (2009) Mortality and functional morbidity after use of PALS/APLS by community physicians. Pediatrics 124: 500-508. [Crossref]

4. Yang CP, Hunt EA, Shilkofski N, Dudas R, Egbuta C, et al. (2016) Can Telemedicine Improve Adherence to Resuscitation Guidelines for Critically Ill Children at Community Hospitals? A Randomized Controlled Trial Using High-Fidelity Simulation. Pediatr Emerg Care 37: 474-479. [Crossref]

5. Harris PA, Taylor R, Thielke R, Payne J, Gonzalez N, et al. (2009) Research electronic data capture (REDCap)-A metadata-driven methodology and workflow process for providing translational research informatics support. J Biomed Inform 42: 377-381. [Crossref]

6. Burke B (2015) Direct-to-consumer telehealth care threatens medical home. APP News 36: 15 .

7. Schwindling L, Ragoschke-Schumm A, Kettner M, Helwig S, Manitz M, et al. (2016) Prehospital Imaging-Based Triage of Head Trauma with a Mobile Stroke Unit: First Evidence and Literature Review. J Neuroimaging 26: 489-493. [Crossref]

8. Gregersen TL, Green A, Frausing E, Ringbæk T, Brøndum E, et al. (2016) Do telemedical interventions improve quality of life in patients with COPD? A systematic review. Int J Chron Obstruct Pulmon Dis 11: 809-822. [Crossref]

9. Hall-Barrow J, Hall RW, Burke BL Jr (2009) Telemedicine and neonatal regionalization of care-ensuring that the right baby gets to the right nursery. Pediatr Ann 38: 557-561. [Crossref]

10. Lim D, Oakley AMM, Rademaker M (2012) Better, sooner, more convenient: A successful teledermoscopy service. Australas J Dermatol 53: 22-25. [Crossref]

11. Marcin JP, Nesbitt TS, Kallas HJ, Struve SN, Traugott CA, et al. (2014) Use of telemedicine to provide pediatric critical care inpatient consultations to underserved rural Northern California. J Pediatr 144: 375-380. [Crossref]

12. Thomas JF, Novins DK, Hosokawa PW, Olson CA, Hunter D, et al. (2018) The use of telepsychiatry to provide cost-efficient care during pediatric mental health emergencies. Psychiatr Serv 69: 161-168. [Crossref] 
Waltzman ML (2018) Bridging the Gap with Telemedicine: Community Emergency Department to Tertiary Care Pediatric Intensive Care

13. Hollander JE, Davis TM, Doarn C, Goldwater JC, Klasko S, et al. (2017) Recommendations from the First National Academic Consortium of Telehealth. Popul Health Manag. [Crossref]
14. Kruse SC, Karem P, Shifflett K (2016) Evaluating barriers to adopting telemedicine worldwide: A systematic review. J Telemed Telecare 24: 4-12. [Crossref]

Copyright: $\ 2018$ Waltzman ML. This is an open-access article distributed under the terms of the Creative Commons Attribution License, which permits unrestricted use, distribution, and reproduction in any medium, provided the original author and source are credited. 